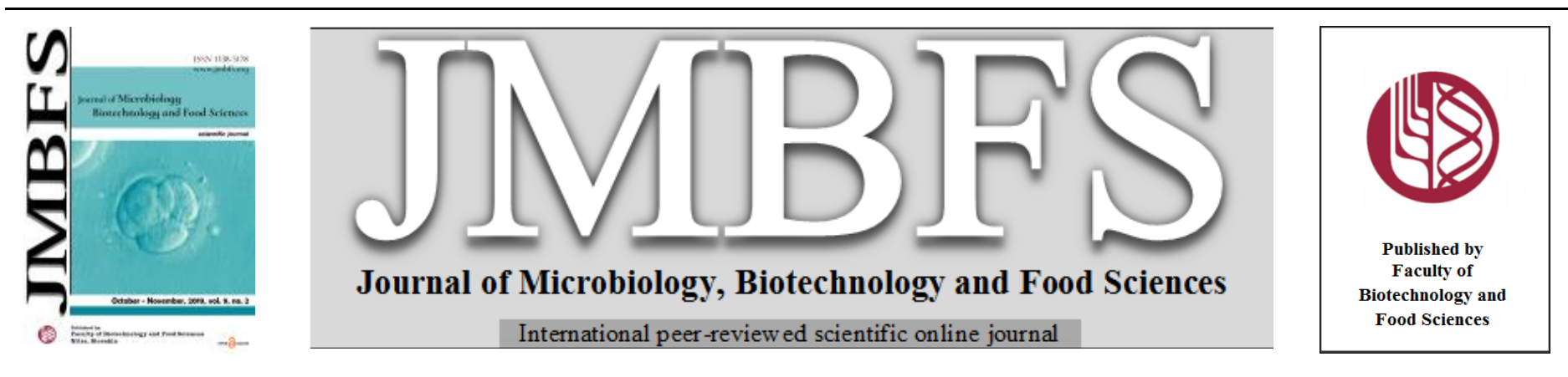

\title{
IN VITRO BIODEGRADATION OF OLEUROPEIN BY LACTOBACILLUS PLANTARUM FSO175 IN STRESS CONDITIONS (pH, NaCl AND GLUCOSE)
}

\author{
Nabil Ghabbour* 1,2, Yahya Rokni ${ }^{1}$, Houssam Abouloifa ${ }^{1}$, Reda Bellaouchi ${ }^{1}$, Nour-Eddine Chihib ${ }^{3}$, Riadh Ben Salah ${ }^{4}, Z^{2}$ hra \\ Lamzira $^{1}$, Ennouamane Saalaoui ${ }^{1}$, and, Abdeslam Asehraou ${ }^{1}$
}

Address(es): Nabil GHABBOUR, PhD,

${ }^{1}$ Laboratory of Biochemistry and Biotechnology, Faculty of Sciences, Mohammed First University, Oujda 60 000, Morocco.

${ }^{2}$ Biotechnology, Environmental Technology and Valorization of Bio-resources team, Faculty of Science and Technology Al-Hoceima. Ajdir 32003, Abdelmalek

Essaâdi University, Morocco.

${ }^{3}$ INRA-UMR UMET 8207 - PIHM team, CNRS-INRA, University of Lille, 369 rue Jules Guesde, BP20039, 59651 Villeneuve-d'Ascq Cedex, France.

${ }^{4}$ Laboratory of Microorganisms and Biomolecules, Center of Biotechnology of Sfax, BP 1177, 3018, Tunisia.

*Corresponding author: n.ghabbour@uae.ac.ma

doi: 10.15414/jmbfs.2020.9.4.769-773

\section{ARTICLE INFO}

Received 11. 12. 2018

Revised 3. 9. 2019

Accepted 11. 9. 2019

Published 3. 2. 2020

Regular article OPEN $\partial_{\text {ACCESS }}$

\begin{abstract}
The objective of this work is to study the oleuropein (OLP) biodegradation by a strain of Lactobacillus plantarum FSO175 under conditions ( $\mathrm{pH} 4.5, \mathrm{NaCl} 5 \%$ and glucose $1 \%$ ), during 7 days of incubation at $30{ }^{\circ} \mathrm{C}$, in modified MRS broth containing OLP as carbon source. The results obtained, by HPLC analyses, showed that the biodegradation of OLP by L. plantarum FSO175, is accompanied with increase of hydroxytyrosol (HT) content and acidity values. The yields of OLP degradation and HT accumulation are depending on carbon sources, stress conditions and time of incubation. So that, the drastic reduction in OLP biodegradation, obtained at $\mathrm{pH} 6.7$ (trial B), decreased significantly $(\mathrm{p}<0.05$ ) in presence of $\mathrm{NaCl} 5 \%$ (trial D) and with glucose $1 \%$ (trial C). Indeed, the OLP biodegradation rate was maximal at $\mathrm{pH} 4.5$ (trial $\mathrm{E}$ ) and was higher even with $\mathrm{NaCl} 5 \%$ (trial F). In contrast, in presence of combined stress conditions (glucose $1 \%, \mathrm{pH} 4.5$ and $\mathrm{NaCl} 5 \%$ : trial G), the biodegradation of OLP decreased significantly ( $<<0.05$ ). The effectiveness of the strain L. plantarum FSO175 in OLP biodegradation leading to variable yields of HT production, revealed its promising perspectives as starter culture, under controlled stress conditions of $\mathrm{pH} 4.5$ and $\mathrm{NaCl} 5 \%$, allowing the production of green table olives rich of HT, the main antioxidant highly desired in foods.
\end{abstract}

Keywords: Biodegradation, Hydroxytyrosol, L. plantarum, Oleuropein, Stress conditions

\section{INTRODUCTION}

Oleuropein (OLP), a secoiridoid glucoside naturally found in leaves and fruits of Olea europaea $\mathrm{L}$., is the major natural phenolic compound responsible for the bitterness in the unripe olives (Othman et al., 2009). In addition to its bitter taste, the OLP has a high antimicrobial activity against gram positive and gramnegative bacteria (Furneri et al., 2002) and against lactic acid bacteria (LAB) mainly Lactobacillus (Juven et al., 1968; Fleming et al., 1973; Rodriguez et al., 2009), the most important microflora highly desired to succeed the lactic fermentation process of green olives (Asehraou et al., 1993). The antimicrobial effect of OLP against lactic acid bacteria affects negatively the fermentation process of green olives by producing the final product with undesirable quality (Hurtado et al., 2012).

To reduce the bitterness and antimicrobial effects of OLP, industrial process for green olives is based on chemicals debittering with the alkali- treatment of fruits, followed by washings and brining to undergo a natural lactic fermentation process (Brenes et al., 1998). However, this chemical debittering leads to high losses in nutrient value of processed olives (Othman et al., 2009), and generates high amounts of spoiled fruits and alkaline waste waters (Kailis et al., 2007). To palliate this chemical debittering inconvenient, the study of OLP biodegradation is essential. The OLP biodegradation leads to the release of glucose, OLP aglycone, elenolic acid and Hydroxytyrosol (HT) (Ciafardini et al., 1994; Marsilio et al., 1996; Rozés et al., 1996; Marsilio et al., 1998; Peres et al., 2014). In a previous work, we have selected some OLP degrading LAB strains, belonging to Lactobacillus, Leuconostoc and Pediococcus species (Ghabbour et al., 2011). Recently, we have reported the effectiveness of Lactobacilli strains to develop a suitable OLP controlled lactic fermentation process of Moroccan Picholine green olives (Ghabbour et al., 2016). The biodegradation of OLP was not well developed in this process, associated with a residual bitter taste in fermented olives, indicating that the biochemical conditions of OLP biodegradation by LAB are not well understood. Furthermore, limited studies were done on OLP biodegradation by LAB in stress conditions close to industrial environment.

The main objective of the present work was to study the biodegradation of OLP by L. plantarum FSO175, previously selected in our laboratory as OLP degrading strain, under the main stress factors $(\mathrm{pH} 4.5, \mathrm{NaCl} 5 \%$ and glucose $1 \%)$ affecting the industrial green table olive process.

\section{MATERIAL AND METHODS}

\section{Chemicals}

The OLP and HT used in this study were purchased from Extrasynthese (Genay, France). The solvents used (ethyl acetate, acetonitrile, methanol and acetic acid) were HPLC Grade and purchased from Sigma Aldrich.

\section{LAB Strain}

The strain of $L$. plantarum FSO175 used in this study was isolated, in our laboratory, from natural fermenting Moroccan green olives. It was selected for its OLP-degrading capacity (Ghabbour et al., 2011), and for its effectiveness in controlled fermentation process of Moroccan Picholine Green Olives (Ghabbour et al., 2016).

\section{Culture Conditions}

The culture assay was conducted on modified de Man, Rogosa and Sharpe (MRS) broth with the following composition: $1 \mathrm{~g} / 1$ of peptone from meat (Biokar, France); $1 \mathrm{~g} / \mathrm{l}$ of yeast extract (Biokar, France); $2 \mathrm{~g} / 1$ of potassium phosphate dibasic (Sigma, Aldrich); 1.5g/l of sodium acetate trihydrate (Sigma, Aldrich); 
$2 \mathrm{~g} / 1$ of triammonium citrate (Sigma, Aldrich); $0.2 \mathrm{~g} / 1$ of magnesium sulfateheptahydrate (Sigma, Aldrich); $0.05 \mathrm{~g} / 1$ of manganese sulfatetetrahydrate (Sigma, Aldrich) and $1 \mathrm{~mL}$ of Tween 80 (Sigma, Aldrich). The carbon sources tested were 1\% (w/v) of OLP (Extrasynthese, Genay, France) and 1\% (w/v) of glucose (Sigma, Aldrich)

The culture trials were conducted on modified MRS media (Table 1) as follows: The trials $\mathrm{A}, \mathrm{B}, \mathrm{C}$ and $\mathrm{D}$ were adjusted to $\mathrm{pH}$ 6.7, and supplemented with: glucose $1 \%$ (Trial A); OLP 1\% (Trial B); OLP $1 \%$ and glucose $1 \%$ (Trial C); OLP $1 \%$ and $\mathrm{NaCl} 5 \%(\mathrm{w} / \mathrm{v})$ (Trial D). The other trials (E, F and G) were acidified to $\mathrm{pH} 4.5$ with $\mathrm{HCl}(0.1 \mathrm{~N})$ and supplemented with OLP $1 \%$ (Trial E); OLP $1 \%$ and $\mathrm{NaCl} 5 \%$ (Trial F); OLP 1\%, glucose $1 \%$ and $\mathrm{NaCl} 5 \%$ (Trial G) All the trials were inoculated with $0.1 \%$ of an overnight culture of $L$ plantarum FSO175, previously cultivated in the same modified MRS broth containing glucose $(1 \%, \mathrm{w} / \mathrm{v})$ as carbon source. Parallel with these trials, assay (trial $\mathrm{H}$ ) containing the same composition as trial $\mathrm{B}$, but without inoculation, was added to check the stability of PLO during this experiment.

The controls used in this experiment were Trial A as positive control and Trial $\mathrm{H}$ as negative control. The positive control, containing $1 \%$ of glucose and inoculated with $L$. plantarum FSO175, was used to observe the normal growth of the strain L. plantarum FSO175; while the negative control (Trial $\mathrm{H}$ ), containing $1 \%$ of OLP and not inoculated, was used to observe the stability of OLP in the experiment conditions.

The assays of a volume of $100 \mathrm{~mL}$, made in triplicate, were incubated at $30^{\circ} \mathrm{C}$ for 7 days. $7 \mathrm{~mL}$ of samples were aseptically collected at $0,1,2,3,5$ and 7 days of incubation for physicochemical and microbiological analyses.

\section{Microbiological Analysis}

The microbial biomass was enumerated using the poor plate technique. $1 \mathrm{~mL}$ of each trial culture suspension was serially diluted $\left(10^{-1}\right.$ to $\left.10^{-10}\right)$ and plated on Petri dish containing MRS Agar. The cultures were incubated at $30{ }^{\circ} \mathrm{C}$ for $24-48 \mathrm{~h}$ and plates containing 30 to 300 colonies were counted and recorded as a colony forming unit per milliliter $(\mathrm{CFU} / \mathrm{mL})$ of culture suspension. The results were expressed as Log CFU/mL.

\section{Physico-Chemical Analysis}

The physico-chemical parameters analyzed were $\mathrm{pH}$, free acidity and reducing sugars. The $\mathrm{pH}$ was measured using a pH-meter type WTW pH 330/SET-1 (VWR) after calibration at $\mathrm{pH} 4$ and 7 . The free acidity was determined by $\mathrm{NaOH}$ $(0.9 \mathrm{~N})$ dosage in presence of phenolphthalein as an indicator. The free acidity was expressed in percent of lactic acid in culture medium $(\%, \mathrm{w} / \mathrm{v})$. The soluble sugars were determined by Ashwell method (Ashwell, 1957), and the results were expressed in percent of glucose $(\%, w / v)$. The method consists of measuring, at $630 \mathrm{~nm}$, the color produced by the reaction of anthrone with the hydrolysis products of soluble sugars released with concentrated sulfuric acid.

\section{Oleuropein and Hydroxytyrosol Analysis}

Samples of microbial culture of $1 \mathrm{~mL}$, collected initially at 0,3 , and 7 days of culture, were used to analyze OLP and HT analyses by an HPLC- DAD system. Samples were centrifuged at $6000 \mathrm{~g}$ during $10 \mathrm{~min}$, and the supernatant was extracted three times with ethyl acetate and then evaporated. The residue obtained was dissolved in $1 \mathrm{~mL}$ of methanol and filtered through PVDF syringe filter (Sartorius, France). Then, a volume of $20 \mu \mathrm{L}$ was injected into HP isocratic LC system, equipped with a HP-UV detector at $280 \mathrm{~nm}$ and a C18 Supelcogel column $(\mathrm{C}-610 \mathrm{H}, 30 \mathrm{~cm} \times 7.8 \mathrm{~mm})$ maintained at $40^{\circ} \mathrm{C}$. The mobile phase consisted of acetonitrile-methanol (1:1, v/v) (solution A) and milli-Q water acidified with acetic acid to $\mathrm{pH} 3.2$ (solution $\mathrm{B}$ ). The flow rate is $1 \mathrm{~mL} / \mathrm{min}$ and the gradient used was the same as described by Mateos et al. (2001). The standards of OLP and HT were dissolved in methanol $(1 \mathrm{~mL})$ and analyzed by the same method described above.

\section{Statistical Analysis}

All the determinations were performed in triplicate. The results were expressed as mean values and standard mean error. The statistical analysis of data was performed by ANOVA using Statgraphics Centurion version XVII. Means values were compared by Least Significant Difference (LSD) test and the significant level was set at $5 \%(\mathrm{p}<0.05)$.

\section{RESULTS}

\section{Physico-Chemical Analysis}

The results of $\mathrm{pH}$ and free acidity obtained were reported on Figures 1 and 2 , respectively. In the media acidified to $\mathrm{pH} 4.5$ (trials $\mathrm{E}, \mathrm{F}$, and $\mathrm{G}$ ), a slight decrease in $\mathrm{pH}$ values was observed, accompanied simultaneously with low increase in free acidity values. No significant difference $(\mathrm{p}<0.05)$ in $\mathrm{pH}$ and free acidity changes were observed between these trials. However, all the assays (trials A, B, C, and
D) initiated at pH 6.7 and containing glucose 1\% (w/v) and/or OLP 1\% (w/v) as carbon sources and inoculated with $L$. plantarum FSO175, showed important and variable acidification rates, with significant differences according to LSD test at $\mathrm{p}$ $<0.05$. This may be due to the carbon source used and the presence or absence of $\mathrm{NaCl} 5 \%$ as the stress factor. Hence, the lowest final $\mathrm{pH}$ value $(\mathrm{pH} 4.7)$ and the highest final free acidity value ( $0.72 \%$ of lactic acid) were obtained with glucose as carbon source (trial A), followed by the combination of glucose and OLP as carbon sources (trial C). The OLP as a sole carbon source (trial B), led to final pH and acidity values of 5.5 and $0.52 \%$, respectively. The use of OLP in presence of $\mathrm{NaCl} 5 \%$ (trial D) led to the lowest acidification rate, with 5.7 and $0.42 \%$ as final values of $\mathrm{pH}$ and free acidity, respectively.

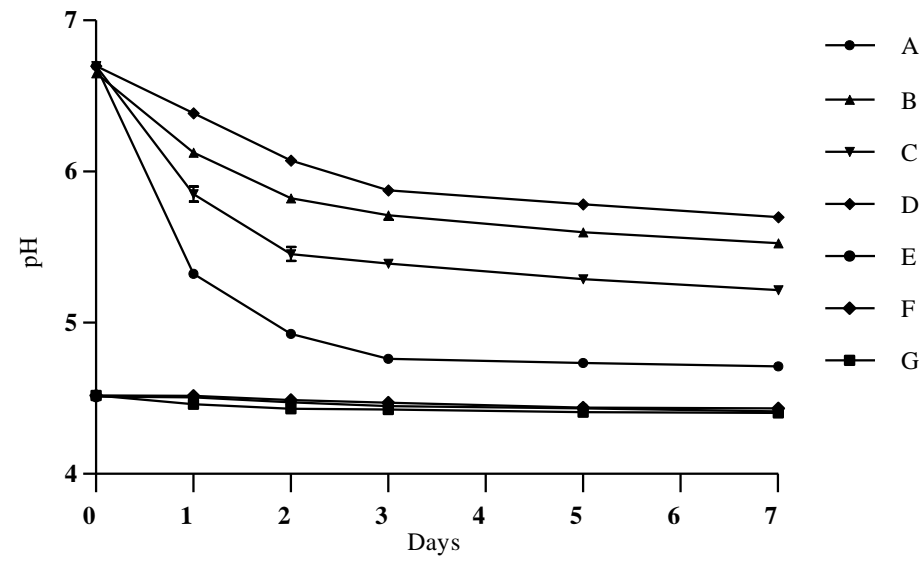

Figure $1 \mathrm{pH}$ changes of L. plantarum FSO175 culture at $30{ }^{\circ} \mathrm{C} / 7$ days in modified MRS mediums supplemented with glucose $1 \%$ (Trial A); OLP 1\% (Trial B); OLP $1 \%$ and glucose $1 \%$ (Trial C); OLP $1 \%$ and $\mathrm{NaCl} 5 \%$ (Trial D), OLP $1 \%$ and pH 4.5 (Trial E); OLP 1\%, NaCl 5\% and pH 4.5 (Trial F); OLP 1\%, glucose $1 \%$, $\mathrm{NaCl} 5 \%$ and $\mathrm{pH} 4.5$ (Trial G).

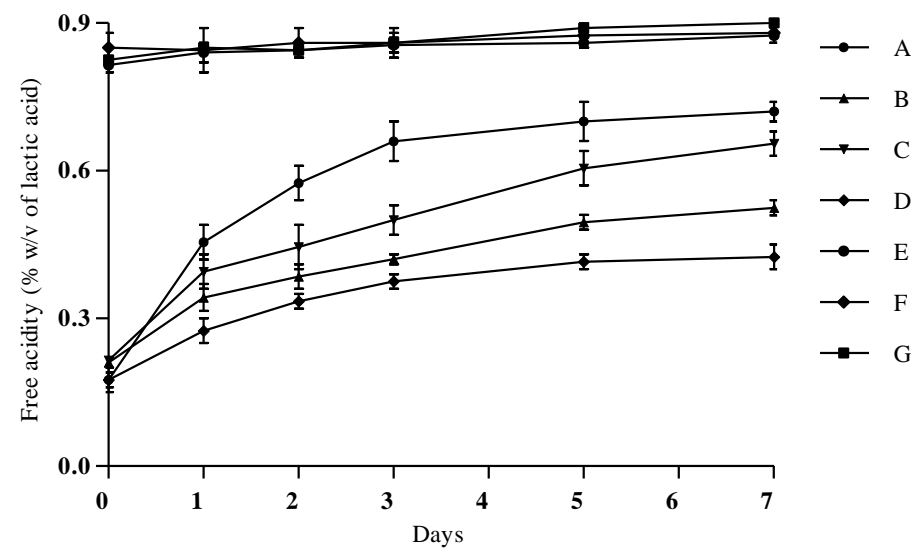

Figure 2 Free acidity changes (\% of lactic acid) of L. plantarum FSO175 culture at $30{ }^{\circ} \mathrm{C} / 7$ days in modified MRS mediums supplemented with glucose $1 \%$ (Trial A); OLP $1 \%$ (Trial B); OLP $1 \%$ and glucose $1 \%$ (Trial C); OLP $1 \%$ and $\mathrm{NaCl} 5 \%$ (Trial D), OLP $1 \%$ and $\mathrm{pH} 4.5$ (Trial E); OLP $1 \%, \mathrm{NaCl} 5 \%$ and $\mathrm{pH} 4.5$ (Trial F); OLP $1 \%$, glucose $1 \%, \mathrm{NaCl} 5 \%$ and $\mathrm{pH} 4.5$ (Trial G).

The sugar contents, expressed in $\%(w / v)$ of glucose, were reported on Figure 3. The results obtained, in trials containing glucose alone or combined with OLP as carbon sources (trials A, C, G), showed a high decrease of sugar contents, from $1 \%$ to $0.12-0.18 \%$, during the first 3 days of culture, followed by a progressive decrease during the last 4 days of culture, with final concentrations of about 0.01 $0.04 \%$. At the end of the experiments, significant differences in glucose content were observed basing LSD test at $\mathrm{p}<0.05$. While, the assays containing OLP as a sole carbon source (trials B, D, E, F) showed a low decrease of glucose from $0.03-0.036 \%$, obtained after 1 day of incubation, to $0.0003-0.007 \%$ at the end of culture. In presence of OLP, the glucose variation showed the same allure in presence or absence of $\mathrm{pH} 4.5$ and/or $\mathrm{NaCl} 5 \%$, but with lower concentrations of glucose, in this condition, the release of glucose linked to OLP and its biodegradation by L. plantarum FSO175 occurs. The comparison of trials B, D, E, and $\mathrm{F}$, at the end of the culture assays, denoted that the difference in glucose content is not statistically significant according to LSD test at $\mathrm{p}<0.05$. 


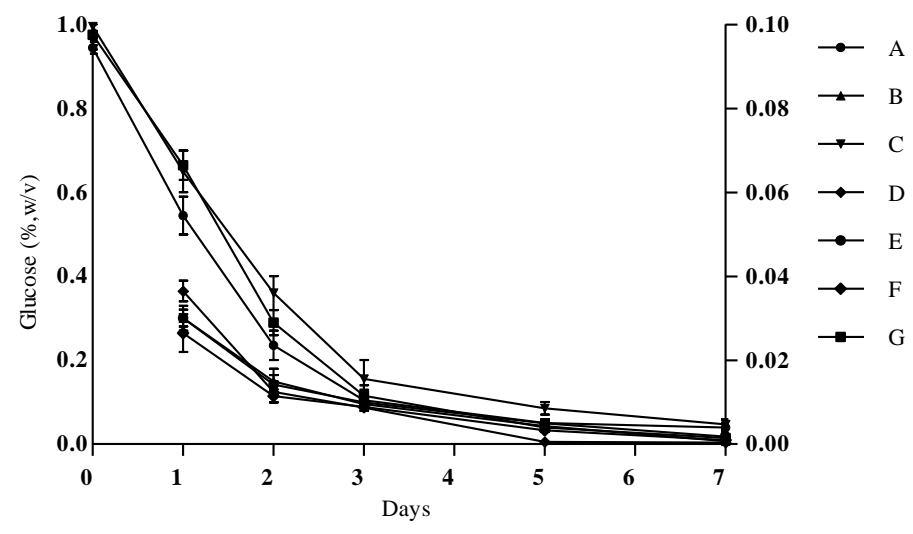

Figure 3 Soluble sugars (\% of glucose) changes of L. plantarum FSO175 culture at $30{ }^{\circ} \mathrm{C} / 7$ days in modified MRS mediums supplemented with glucose $1 \%$ (Trial A); OLP $1 \%$ (Trial B); OLP 1\% and glucose $1 \%$ (Trial C); OLP 1\% and $\mathrm{NaCl} 5 \%$ (Trial D), OLP $1 \%$ and $\mathrm{pH} 4.5$ (Trial E); OLP $1 \%, \mathrm{NaCl} 5 \%$ and $\mathrm{pH} 4.5$ (Trial F); OLP $1 \%$, glucose $1 \%, \mathrm{NaCl} 5 \%$ and $\mathrm{pH} 4.5$ (Trial G). Left $\mathrm{Y}$ axis (\% of glucose, w/v) for trials A, C, and G. Right Y axis (\% of glucose, w/v) for trials B, D, E and

\section{Microbiological Analysis}

All the inoculated trials showed increase in biomass of L. plantarum FSO175 in presence of glucose and/or OLP as carbon sources (Figure 4). The biomass growth depended on carbon sources and stress conditions. Significant differences $(\mathrm{p}<0.05)$ in biomass growth were obtained between all the culture assays. The higher final biomass values were obtained with glucose alone $(9.285 \log \mathrm{CFU} / \mathrm{mL}$, Trial A) or combined with OLP (9.207 log CFU/mL, Trial C) as carbon sources. In presence of OLP as a sole carbon source, the biomass values obtained were lower, and were $9.151 \log \mathrm{CFU} / \mathrm{mL}$ in the absence of stress factors (trial B), $9.004 \log \mathrm{CFU} / \mathrm{mL}$ in presence of $\mathrm{NaCl} 5 \%$ (trial D), and $8.925 \log \mathrm{CFU} / \mathrm{mL}$ in presence of $\mathrm{pH} 4.5$ (trial E).

Table 1 Means values of OLP, HT changes ([\%, w/v] \pm ESM) and yields (\%) of OLP biodegradation and HT production, of $L$. plantarum $\mathrm{FSO} 175$ culture at $30{ }^{\circ} \mathrm{C} / 7$ days in modified MRS mediums supplemented with glucose 1\% (Trial A); OLP 1\% (Trial B); OLP 1\% and glucose 1\% (Trial C); OLP 1\% and NaCl 5\% (Trial D), OLP $1 \%$ and pH 4.5 (Trial E); OLP $1 \%, \mathrm{NaCl} 5 \%$ and pH 4.5 (Trial F); OLP $1 \%$, glucose $1 \%, \mathrm{NaCl} 5 \%$ and $\mathrm{pH} 4.5$ (Trial G).

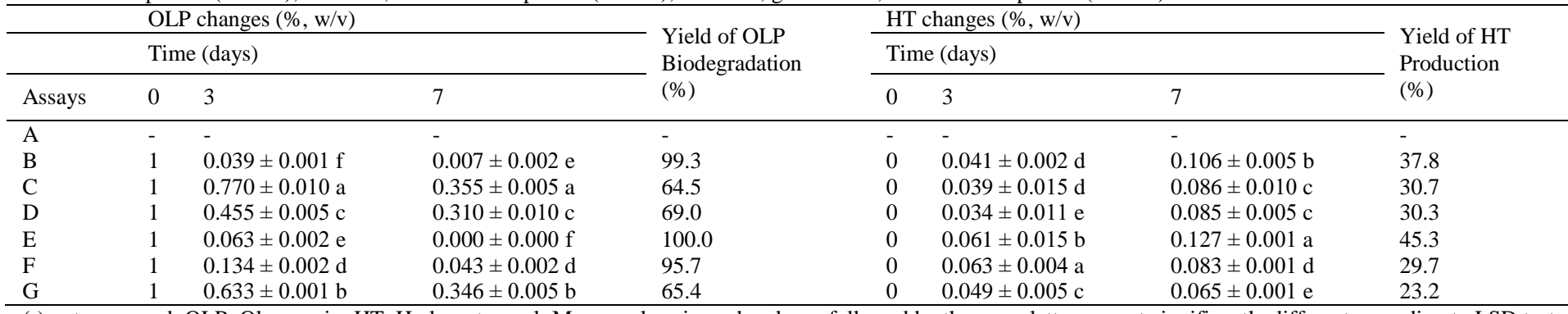

(-) not measured. OLP: Oleuropein, HT: Hydroxytyrosol. Means values in each column followed by the same letter are not significantly different according to LSD test at $\mathrm{p}<0.05$.

The results of OLP and HT changes, obtained in all inoculated assays, showed that the increment of OLP biodegradation doesn't correspond to that of HT production (Table1). Looking for the results in trial $\mathrm{B}$, in the first period (first 3 days) of culture assay, $0.961 \mathrm{~g} \%$ of OLP corresponding to $1.78 \mathrm{mM}$ were totally hydrolyzed and yielded low HT content of $0.041 \%(0.266 \mathrm{mM})$. Whereas, in the second period (last 4 days) of culture assay, only $0.032 \mathrm{~g} \%$ of OLP $(0.06 \mathrm{mM})$ was hydrolyzed and produced high HT content of $0.065 \%(0.423 \mathrm{mM})$, which means that in this period, an amount of OLP was 30 times lower than that hydrolyzed in the first period, have produced an amount even greater of HT as compared to that produced in the first period. This finding may lead us to conclude that the OLP biodegradation occurs at least in two steps. The first step of OLP Biodegradation generated the intermediate compounds of HT production, which undergo second step of bioconversion giving rise to high content of HT.

In presence of OLP as a sole carbon source, the OLP decrease and HT increase were higher in the absence of stress factors (trial B), at $\mathrm{pH} 4.5$ (trial E), and with the combination of $\mathrm{pH} 4.5$ and $\mathrm{NaCl} 5 \%$ (trial F), with $0.007 \%, 0 \%$ (not detected) and $0.043 \%$ as final OLP concentrations, respectively (Table 1). The HT final concentrations obtained in these conditions were $0.106 \%, 0.127 \%$ and $0.083 \%$, respectively in trials B, E and F. The highest OLP biodegradation rate $(100 \%)$ was obtained at $\mathrm{pH} 4.5$ (trial E), leading to the maximum HT production rate $(45.3 \%)$. Significant difference (at p <0.05) in OLP and HT changes were obtained between these trials (Table 1 ).

In presence of $\mathrm{NaCl} 5 \%$ (trial D), the decrease of OLP was significantly lower, and its final concentration obtained was $0.31 \%$, associated with the HT

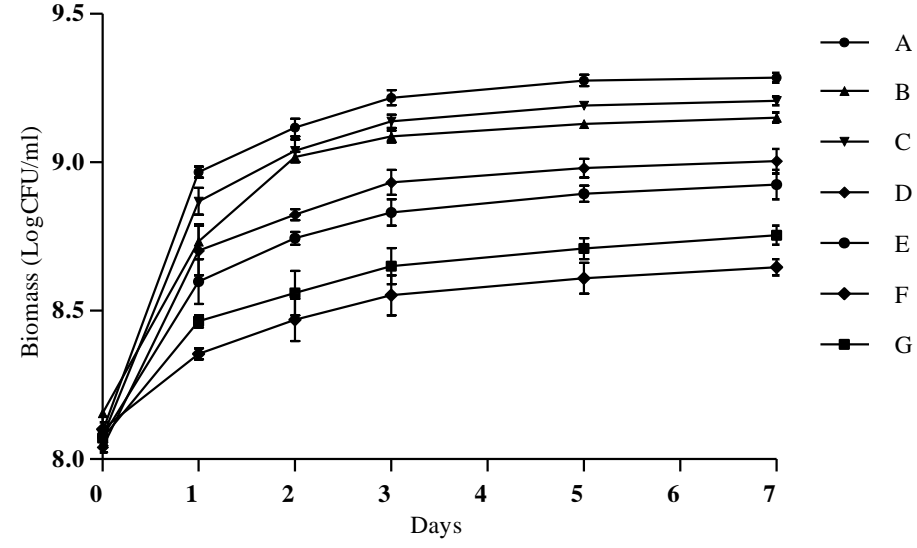

Figure 4 Biomass growth changes ( $\log \mathrm{CFU} / \mathrm{mL}$ ) of $L$. plantarum FSO175 culture at $30{ }^{\circ} \mathrm{C} / 7$ days in modified MRS mediums supplemented with glucose 1\% (Trial A); OLP 1\% (Trial B); OLP 1\% and glucose 1\% (Trial C); OLP $1 \%$ and $\mathrm{NaCl} 5 \%$ (Trial D), OLP $1 \%$ and $\mathrm{pH} 4.5$ (Trial E); OLP $1 \%$, $\mathrm{NaCl} 5 \%$ and $\mathrm{pH} 4.5$ (Trial F); OLP $1 \%$, glucose $1 \%, \mathrm{NaCl} 5 \%$ and $\mathrm{pH} 4.5$ (Trial $\mathrm{G})$.

\section{Oleuropein and Hydroxytyrosol Measurements}

The OLP, HT contents (\%, w/v) and yields of OLP biodegradation and HT production $(\%)$ are reported on Table 1 . These results showed a drastic reduction of OLP biodegradation, in all inoculated trials (B, C, D, E, F and G), from $1 \%$ (initial concentration) to values ranging between $0 \%$ and $0.355 \%$, corresponding to yield of OLP biodegradation between $64.5 \%$ and $100 \%$. The drastic bioconversion of OLP was accompanied with the concomitant accumulation of HT, with the final concentrations ranging between $0.065 \%$ and $0.127 \%$, corresponding to yield of HT production between $23.2 \%$ and $45,3 \%$. While, in non-inoculated assay (trial $\mathrm{H}$ ), the OLP was stable during the 7 days of culture at $30{ }^{\circ} \mathrm{C}$. accumulation of $0.085 \%$. The lowest decrease of OLP was obtained in presence of glucose, showing $0.355 \%$ and $0.346 \%$ as final concentrations of OLP, in absence (trial C) and in presence of stress conditions (trial G), respectively. Thus, the HT final concentrations resulted were 0.086 and $0.065 \%$ respectively in trials C and G (Table 1). These results indicate that the biodegradation of OLP reduced significantly in presence of glucose, which is traduced by significant difference in HT production. Comparing the result of OLP and HT changes in trails C and G (Table 1), we conclude that, the combined stress factors ( $\mathrm{pH} 4.5$ and $\mathrm{NaCl} 5 \%$ ) affected positively the OLP biodegradation, but in the opposite, they are affecting negatively the yield of HT production. This finding indicates that the pathways of OLP biodegradation and/or their mechanism are depending on the stress conditions present in the media.

\section{DISCUSSION}

L. plantarum FSO175 was isolated from naturally fermented non-alkali-treated Moroccan Picholine green olives, and was selected for its tolerance and biodegradation capacity of OLP in the absence of stress factors (Ghabbour et al., 2011). This strain showed its effectiveness in developing controlled lactic fermentation process of no-debittered Green Olives (Ghabbour et al., 2016) However, the fermented olives remain with bitter taste, indicating a lack of oleuropein biodegradation process. In the present work, we have studied the biodegradation of OLP by L. plantarum FSO175 in the presence of the main 
stress factors affecting the lactic fermentation process of green olives, including $\mathrm{pH} 4.5, \mathrm{NaCl} 5 \%$ and glucose $1 \%$ as carbon source.

The results obtained showed that acidification rate, degradation of glucose and biomass growth of $L$. plantarum FSO175, were higher in presence of glucose than those obtained with glucose combined with OLP as a carbon source. This finding indicates the inhibitory effect of OLP against LAB growth, which was already reported by several authors (Juven et al., 1968; Fleming et al., 1973; Rodriguez et al., 2009), while, other authors reported its bactericidal effect against other LAB strains (Ruiz-Barba et al., 1991).

The glucose contents obtained, in all assays containing OLP as a sole carbon source in different stress conditions (trials B, D, E, F), are released as a result of $\beta$-glucosidase activity, an inducible enzyme previously demonstrated by L. plantarum in presence of OLP as a sole carbon source (Landete et al., 2008). The decrease of glucose during incubation time is due to its metabolism by $L$. plantarum FSO175, traduced by the decrease in $\mathrm{pH}$ and increase of acidity. As reported by Rozés et al. (1996), the aciditfication rate obtained in all assays containing OLP as a sole carbon source may be attributed to the accumulation of lactic acid. Basing on these results, we may conclude about the effectiveness of L. plantarum FSO175 to grow on OLP as carbon source and in presence of various stress factors. The tolerance and biodegradation capacity of OLP by $L$ plantarum FSO175 in these stress conditions could be due to its natural selection during spontaneous fermentation process of non- alkali-treated green olives.

The biodegradation of OLP was reported for L. plantarum (Ciafardini et al., 1994; Ghabbour et al., 2011; Zago et al., 2013; Kaltsa et al., 2015; Ghabbour et al., 2016; Ramírez et al., 2017). In this work we confirmed the in vitro OLP biodegradation capacity of L. plantarum FSO175 in presence of different stress conditions. The OLP contents decreased significantly $(\mathrm{p}<0.05)$ in all the assays for 7 days of culture, but with variable yields of biodegradation (Table 1). The disagreements in OLP biodegradation and HT accumulation, observed in all culture assays, may lead us to conclude that the OLP biodegradation occurs at least in two steps. During the first period ( 3 days) of culture, the highest biodegradation of OLP by L. plantarum FSO175, should be attributed, mainly to $\beta$-glucosidase and partially to esterase enzymes. In that period, the $\beta$-glucosidase gives rise to glucose and OLP aglycone as an intermediate compound of HT production (Tuna et al., 2009), the esterase gives rise to HT and elenolic acid glucoside (Segovia-Bravo et al., 2009), this explanation justifies clearly the highest OLP decrease and lowest accumulation of HT. However, the high amount of HT obtained during the second period (last 4 days) of culture, could not be yielded from the biodegradation of low remained content of OLP, but could be provided from the second bioconversion of an intermediate compound of HT production, OLP aglycone, by means of esterase action, giving rise to HT and elenolic acid (Marsilio et al., 1996; Marsilio et al., 1998; Segovia-Bravo et al., 2009).

The OLP biodegradation rate obtained in the absence of glucose, as a second carbon source, and stress conditions ( $\mathrm{pH} 4.5$ and $\mathrm{NaCl} 5 \%$ ) were higher and associated with biomass growth and acidification of the medium. In this condition, the yield of HT production of $37.8 \%$ obtained with $L$. plantarum FSO175 is higher than those of 25\% and 30\%, reported by Marsilio et al. (1998), and Santos et al. (2012) respectively. However, the lowest biodegradation rate was obtained in presence of glucose as a second carbon source in absence or presence of stress conditions ( $\mathrm{pH} 4.5, \mathrm{NaCl} 5 \%$ ). The presence of glucose reduced significantly the OLP biodegradation rate even with important biomass growth. This result may be explained by the inhibition of $\beta$ glucosidase production and/or activity of $L$. plantarum FSO175 by glucose $1 \%$. These findings are in agreement with (Elshafei et al., 2011) who reported the inhibition of $\beta$-glucosidase activity by glucose.

The results obtained showed that the OLP biodegradation capacity of $L$. plantarum FSO175 is significantly reduced in presence of $\mathrm{NaCl} 5 \%$, which is explained by the significant decrease obtained in biomass growth. This finding is in according to that reported that the addition of both $\mathrm{NaCl}$ and OLP decreased stronglythe growth and survival of L. plantarum ((Rozés et al., 1996). However, the highest OLP biodegradation $(100 \%)$ was obtained at $\mathrm{pH} 4.5$ with the maximum yield of HT production of $45.3 \%$. Even with combined conditions ( $\mathrm{pH} 4.5$ and $\mathrm{NaCl} 5 \%$ ) and low biomass growth, the OLP biodegradation was higher. This result may be due to the optimum $\mathrm{pH}$ of $\beta$-glucosidase activity, which is near of pH 4-5 (Elshafei et al., 2011; Wei et al., 2011). The OLP is stable in acid conditions (Gikas et al., 2006) and is not degraded by the acid environments created by fermentation (Kailis et al., 2007). A slow acid hydrolysis of OLP can occur at lower $\mathrm{pH}$ (Capasso et al., 1997) than the $\mathrm{pH} 4.5$ we used in this experiment. Furthermore, the acid hydrolysis of OLP cannot release significant amounts of HT in comparison to the enzymatic biodegradation (Khoufi et al., 2011). All these arguments indicate that the biodegradation of OLP in our cultures couldn't be attributed to acidic effect, but mainly due to the oleuropeinase activity of enzymes produced by L. plantarum FSO175.

The biodegradation of OLP by $L$. plantarum involving $\beta$-glucosidase and esterase enzymes was reported (Rodriguez et al., 2009; Kaltsa et al., 2015; De Leonardis et al., 2016). Furthermore, LAB with $\beta$-glucosidase and esterase activities are very recommended for table olives processing by assuring the biological debittering of olives instead of chemical process (Ramírez et al., 2017). Our results revealed the promising application of $L$. plantarum FSO175 as a starter, in controlled stress conditions ( $\mathrm{pH} 4.5$ and $\mathrm{NaCl} 5 \%$ ), for debittering and fermenting green olives. Indeed, this process, associated with the accumulation of HT, could lead to desirable safety and nutritional values of fermented Moroccan green table olives.

Indeed, the HT is not commercially available in high amounts as a food additive. Several methods have been proposed for the production of HT by means of chemical or enzymatic synthesis (Capasso et al., 1997; Espín et al., 2001; Khoufi et al., 2011). The results of OLP biodegradation by L. plantarum FSO175 under different conditions ( $\mathrm{pH} 4.5, \mathrm{NaCl} 5 \%$ and glucose $1 \%$ ), leading mainly to the production of HT, as the mean end product of OLP hydrolysis, can be considered as a new perspective for the biological production of HT basing L. plantarum FSO175.

\section{CONCLUSION}

This work demonstrated that the biodegradation of OLP (1\%) as a sole carbon source, by L. plantarum FSO175, under conditions ( $\mathrm{pH} \mathrm{4.5,} \mathrm{NaCl} 5 \%$ and glucose $1 \%$ ), was associated with increases of HT content and acidity value. The tolerance and biodegradation capacity of OLP by L. plantarum FSO175 under these conditions should be due to its natural selection during spontaneous fermentation process of non-alkali-treated green olives. The yields of OLP biodegradation and HT production are depending on the stress conditions, carbon source and incubation time. At $\mathrm{pH} 6.7$ the biodegradation of OLP is higher and decreased significantly in presence of $\mathrm{NaCl} 5 \%$. At $\mathrm{pH} 4.5$ the biodegradation of OLP is maximal with the highest yield of HT production, and is higher even in presence of $\mathrm{NaCl} 5 \%$ and with low biomass growth. In presence of glucose, the OLP biodegradation was reduced significantly even with high biomass growth The effect of $\mathrm{NaCl} 5 \%$ and glucose $1 \%$ on the OLP biodegradation decrease was traduced by significant decrease in acidification rates. The highest oleuropeinolitic activity of L. plantarum FSO175 demonstrated, in presence of combined stress conditions ( $\mathrm{pH} 4.5$ and $\mathrm{NaCl} 5 \%$ ) even with low biomass growth, may be attributed to the optimal conditions of production and/or activity of the main enzymes implicated in OLP bioconversion. The effectiveness of $L$ plantarum FSO175 strain in OLP biodegradation and HT production, revealed its promising perspectives as starter culture: firstly, for bioprocessing of green table olives, under controlled stress conditions of $\mathrm{pH} 4.5$ and $\mathrm{NaCl} 5 \%$, allowing the production of end product rich of $\mathrm{HT}$, the main antioxidant highly desired in foods. And secondly, for biological production of HT from olive mill wastewater.

Acknowledgments: This research was supported by the CNRST under grant N ${ }^{\circ} \mathrm{PPR} / 19 / 2015$, and the Morocco-Tunisia bilateral cooperation (17 TM 06).

\section{REFERENCES}

Asehraou, A., Faid, M., \& Akhartouf, R. (1993). Pure culture fermentation of green olives by lactobacilli strains. Microbiologie-Aliments-Nutrition, 11, 221 228.

Ashwell, G. (1957). Colorimetric analysis of sugars. Methods in Enzymology, 3, 73-105.

Brenes, M., \& de Castro, A. (1998). Transformation of oleuropein and its hydrolysis products during Spanish-style green olive processing. Journal of the

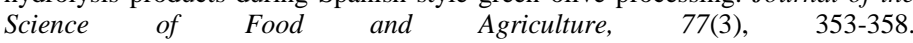
https://doi.org/10.1002/(SICI)1097-0010(199807)77:3<353::AID-

\section{JSFA50>3.0.CO;2-G}

Capasso, R., Evidente, A., Visca, C., Gianfreda, L., Maremonti, M., \& Greco, G. (1997). Production of glucose and bioactive aglycone by chemical and enzymatic hydrolysis of purified oleuropein from Olea europea. Applied Biochemistry and Biotechnology, 61(3), 365-377. https://doi.org/10.1007/BF02787808

Ciafardini, G., Marsilio, V., Lanza, B., \& Pozzi, N. (1994). Hydrolysis of oleuropein by Lactobacillus plantarum strains associated with olive fermentation. Applied and Environmental Microbiology, 60(11), 4142-4147.

De Leonardis, A., Testa, B., Macciola, V., Lombardi, S. J., \& Iorizzo, M. (2016). Exploring enzyme and microbial technology for the preparation of green table olives. European Food Research and Technology, 242(3), 363-370 https://doi.org/10.1007/s00217-015-2546-3

Elshafei, A. M., Hassan, M. M., Morsi, N. M., \& Elghonamy, D. H. (2011) Purification and some kinetic properties of b-glucosidase from Aspergillus terreus NRRL 265. African Journal of Biotechnology, 10(84), 19556-19569. https://doi.org/10.5897/AJB10.2617

Espín, J. C., Soler-Rivas, C., Cantos, E., Tomás-Barberán, F. A., \& Wichers, H. J. (2001). Synthesis of the antioxidant hydroxytyrosol using tyrosinase as biocatalyst. Journal of Agricultural and Food Chemistry, 49(3), 1187-1193. https://doi.org/10.1021/jf001258b

Fleming, H. P., Walter, W. M., \& Etchells, J. L. (1973). Antimicrobial Properties of Oleuropein and Products of Its Hydrolysis from Green Olives. Applied Microbiology, 26(5), 777-782.

Furneri, P. M., Marino, A., Saija, A., Uccella, N., \& Bisignano, G. (2002). In vitro antimycoplasmal activity of oleuropein. International Journal of Antimicrobial Agents, 20(4), 293-296. https://doi.org/10.1016/S09248579(02)00181-4 
Ghabbour, N., Lamzira, Z., Thonart, P., Cidalia, P., Markaoui, M., \& Asehraou, A. (2011). Selection of oleuropein-degrading lactic acid bacteria strains isolated from fermenting Moroccan green olives. Grasas y Aceites, 62(1), 84-89. https://doi.org/10.3989/gya.055510

Ghabbour, N., Rokni, Y., Lamzira, Z., Thonart, P., Chihib, N., Peres, C., \& Asehraou, A. (2016). Controlled fermentation of Moroccan picholine green olives by oleuropein-degrading Lactobacilli strains. Grasas y Aceites, 67(2), e138. http://dx.doi.org/10.3989/gya.0759152

Gikas, E., Papadopoulos, N., \& Tsarbopoulos, A. (2006). Kinetic study of the acidic hydrolysis of oleuropein, the major bioactive metabolite of olive oil Journal of Liquid Chromatography \& Related Technologies, 29(4), 497-508. https://doi.org/10.1080/10826070500474113

Hurtado, A., Reguant, C., Bordons, A., \& Rozes, N. (2012). Lactic acid bacteria from fermented table olives. Food Microbiology, 31(1), 1-8 https://doi.org/10.1016/j.fm.2012.01.006

Juven, B., Samish, Z., \& Henis, Y. (1968). Identification of oleuropein as a natural inhibitor of lactic fermentation of green olives. Journal of Agriculture Research, 18, 137 - 138

Kailis, S., \& Harris, D. J. (2007). Producing table olives (L. Press Ed.). Australia: Landlinks Press.

Kaltsa, A., Papaliaga, D., Papaioannou, E., \& Kotzekidou, P. (2015) Characteristics of oleuropeinolytic strains of Lactobacillus plantarum group and influence on phenolic compounds in table olives elaborated under reduced salt $\begin{array}{llll}\text { conditions. } & \text { Food } & \text { Microbiology, } & \text { 48, }\end{array}$ http://dx.doi.org/DOI:10.1016/j.fm.2014.10.016

Khoufi, S., Hamza, M., \& Sayadi, S. (2011). Enzymatic hydrolysis of olive wastewater for hydroxytyrosol enrichment. Bioresour Technol, 102(19), 90509058. http://dx.doi.org/10.1016/j.biortech.2011.07.048

Landete, J. M., Curiel, J. A., Rodríguez, H., de las Rivas, B., \& Munoz, R. (2008). Study of the inhibitory activity of phenolic compounds found in olive products and their degradation by Lactobacillus plantarum strains. Food Chemistry, 107(1), 320-326. https://doi.org/10.1016/j.foodchem.2007.08.043

Marsilio, V., \& Lanza, B. (1998). Characterisation of an oleuropein degrading strain of Lactobacillus plantarum. Combined effects of compounds present in olive fermenting brines (phenols, glucose and $\mathrm{NaCl}$ ) on bacterial activity. Journal of the Science of Food and Agriculture, 76(4), 520-524. http://dx.doi.org/10.1002/(SICI)1097-0010(199804)76:4<520::AID-

JSFA982>3.0.CO;2-I

Marsilio, V., Lanza, B., \& Pozzi, N. (1996). Progress in table olive debittering: Degradationin vitro of oleuropein and its derivatives by Lactobacillus plantarum Journal of the American Oil Chemists' Society, 73(5), 593-597. https://doi.org/10.1007/BF02518113

Mateos, R., Espartero, J. L., Trujillo, M., Ríos, J. J., León-Camacho, M., Alcudia, F., \& Cert, A. (2001). Determination of Phenols, Flavones, and Lignans in Virgin Olive Oils by Solid-Phase Extraction and High-Performance Liquid Chromatography with Diode Array Ultraviolet Detection. Journal of Agricultural and Food Chemistry, 49(5), 2185-2192. https://doi.org/10.1021/jf0013205

Othman, N. B., Roblain, D., Chammen, N., Thonart, P., \& Hamdi, M. (2009) Antioxidant phenolic compounds loss during the fermentation of Chétoui olives. Food Chemistry, 116(3), 662-669. https://doi.org/10.1016/j.foodchem.2009.02.084

Peres, C. M., Alves, M., Hernandez-Mendoza, A., Moreira, L., Silva, S., Bronze, M. R., . . Malcata, F. X. (2014). Novel isolates of lactobacilli from fermented Portuguese olive as potential probiotics. LWT - Food Science and Technology, 59(1), 234-246. https://doi.org/10.1016/j.lwt.2014.03.003

Ramírez, E., Medina, E., García, P., Brenes, M., \& Romero, C. (2017) Optimization of the natural debittering of table olives. LWT - Food Science and Technology, 77, 308-313. https://doi.org/10.1016/j.1wt.2016.11.071

Rodriguez, H., Curiel, J. A., Landete, J. M., de las Rivas, B., Lopez de Felipe, F., Gomez-Cordoves, C., . . . Munoz, R. (2009). Food phenolics and lactic acid bacteria. International journal of food microbiology, 132(2-3), 79-90. http://dx.doi.org/10.1016/j.ijfoodmicro.2009.03.025

Rozés, N., \& Peres, C. (1996). Effect of oleuropein and sodium chloride on viability and metabolism of Lactobacillus plantarum. Applied Microbiology and Biotechnology, 45(6), 839-843. http://dx.doi.org/10.1007/s002530050771

Ruiz-Barba, J., Garrido-Fernandez, A., \& Jimenez-Diaz, R. (1991). Bactericida action of oleuropein extracted from green olives against Lactobacillus plantarum Letters in Applied Microbiology, 12(2), 65-68. http://dx.doi.org/10.1111/j.1472765X.1991.tb00505.x

Santos, M. M., Piccirillo, C., Castro, P. M., Kalogerakis, N., \& Pintado, M. E (2012). Bioconversion of oleuropein to hydroxytyrosol by lactic acid bacteria World Journal of Microbiology and Biotechnology, 28(6), 2435-2440. https://doi.org/10.1007/s11274-012-1036-Z

Segovia-Bravo, K. A., Jarén-Galán, M., García-García, P., \& Garrido-Fernández, A. (2009). Browning reactions in olives: Mechanism and polyphenols involved. Food Chemistry, $114(4)$, 1380-1385. https://doi.org/10.1016/j.foodchem.2008.11.017

Tuna, S., \& Akpinar-Bayizit, A. (2009). The Use of $\beta$-Glucosidase Enzyme in Black Table Olives Fermentation. Notulae Botanicae Horti Agrobotanici ClujNapoca, 37(2), 182-189. https://doi.org/10.15835/nbha3723145
Wei, R. R., Hughes, H., Boucher, S., Bird, J. J., Guziewicz, N., Van Patten, S. M., . . . Edmunds, T. (2011). X-ray and biochemical analysis of N370S mutant human acid $\beta$-glucosidase. Journal of Biological Chemistry, 286(1), 299-308. https://doi.org/10.1074/jbc.M110.150433

Zago, M., Lanza, B., Rossetti, L., Muzzalupo, I., Carminati, D., \& Giraffa, G. (2013). Selection of Lactobacillus plantarum strains to use as starters in fermented table olives: Oleuropeinase activity and phage sensitivity. Food Microbiology, 34(1), 81-87. https://doi.org/10.1016/j.fm.2012.11.005 